\title{
A comparison of drug treatment outcome of irritable bowel syndrome patients with and without education: a randomized controlled study
}

\author{
B Paik ${ }^{1}$, SK Sarker ${ }^{2}$, PK Chowdhury ${ }^{3}$, MS Ahmed ${ }^{4}$
}

\begin{abstract}
The objective of the prospective randomized controlled comparative study to explore about the role of education in the treatment of irritable bowel syndrome (IBS), a chronic continuous or remittent gastrointestinal illness characterized by frequent unexplained symptoms that include abdominal pain, bloating and bowel disturbance. The patients who fulfilled the set criteria of this study and had normal physical examination were considered to undergo screening investigations ( $\mathrm{Hb} \%$, TC, DC, ESR, blood glucose, serum TSH, stool for R/M/E and short colonoscopy or double contrast barium enema) to exclude any organic disease. In order to administer the same pharmacological treatment only diarrhea predominant patients without any organic disease were recruited for the present study. The number of recruited patients was 80 . Of them, 40 patients were given only pharmacological management with Mebeverine Hydrochloride $135 \mathrm{mg}$ thrice daily half an hour before meal and Amitryptyline $10 \mathrm{mg}$ at night for 6 months, and the other 40 patients were given education by a structured and planned educational class for $1 \mathrm{hr}$ in addition to the same pharmacological treatment. In both groups, changes of symptoms and quality of life of the patients were assessed by using a valid IBS related quality of life (IBS-QOL) instrument. The results showed that significant improvement occurred in both the groups of patients at 1 month and 6 months in respect to their baseline IBS-QOL score. But the improvement was not significantly higher in the group with education in comparison to the group without education. Therefore, it can be concluded that the educational class had no extra impact on drug treatment protocol for IBS used in the present study.
\end{abstract}

Key words: Irritable bowel syndrome (IBS), drug treatment, role of education, IBS-QOL.

\section{Introduction}

Functional gastrointestinal disorders are very common in the society. Irritable bowel syndrome (IBS) and non-ulcer dyspepsia are the most common disorders encountered by gastroenterologists and constitute a considerable economic burden to the health care system. ${ }^{1}$ IBS is a chronic continuous or remittent gastrointestinal illness charac- terized by frequent unexplained symptoms that include abdominal pain, bloating and bowel disturbance, which may be either diarrhea or constipation or an erratic bowel habit that has features of both.

The exact prevalence of IBS in Bangladesh is not known. Estimated prevalence of IBS in one study reported to be $16.9 \%$ among

1. B Paik, Associate Professor, Dept. of Gastroenterology, Khulna Medical College, Khulna. Email: paikbishnu1971@yahoo.com

2. SK Sarker, Associate Professor, Dept. of Gastroenterology, SBMCH, Barisal

3. PK Chowdhury, Assistant Professor, Dept. of Respiratory Medicine, Khulna Medical College, Khulna

4. MS Ahmed, Associate Professor (cc) of Radiology \& Imaging, Kushtia Medical College, Kushtia 
patients attending the out-patient department of a tertiary level hospital. ${ }^{2} \mathrm{~A}$ population based study showed the prevalence of IBS was $20.6 \%$ in male and $27.7 \%$ in female in a rural community of Bangladesh. $^{3}$

Despite much research, the pathophysiology of IBS remains poorly understood. ${ }^{4}$ So, it is very difficult to treat patients with IBS. There is no standard treatment for IBS. Alleviating symptoms is one of the primary challenging goals of caring for IBS patients. Various pharmacological agents are available for treating IBS. The commonly used drugs are bulking agents, anti-diarrheal agents, anti-spasmodics and anti-depressant drugs. Very few drugs have proven to be of value in the management of IBS. A considerable placebo effect and methodological problem in recording efficacy make drug evaluation difficult. ${ }^{5}$ In a review, Klein concluded that there is no therapeutic trial that ever demonstrated the efficacy of any drug in the treatment of IBS. ${ }^{6}$

Because IBS symptoms can be elicited or exacerbated by diet and stress, and this suggests that patient education regarding the illness may be beneficial to the patients in managing their symptoms and thus may be beneficial to the society by improving self-management and decreasing health care utilization. ${ }^{7}$ This has shown to be true for other chronic diseases such as asthma and heart disease. ${ }^{8}$ However, little is known about the role of education in IBS. Therefore, the aim and objective of the present study was to study the effects of outpatient education on the short and long term outcomes of IBS patients in relation to change of symptom and quality of life.

\section{Materials and Method}

This is a prospective randomized controlled comparative study. The patients with abdominal pain/discomfort with altered bowel habit and visited the out-patient department of Gastroenterology Unit, Bangabandhu Sheikh Mujib Medical University (BSMMU) were invited to participate in the present study.
The inclusion criteria included the persons diagnosed as having IBS on the basis of Rome II criteria, must not having any red flag signs, age from 15 to 60 years and having minimum primary education level and who could fill up the questionnaire.

The inclusion criteria included the conditions that may affect the ability of the patient to fill up the questionnaire e.g. dementia, mental retardation, psychosis, blindness, etc; pregnant patients and women who breast feed their child and patients with concomitant severe illness like severe asthma, heart failure, renal disease or chronic liver disease were excluded because of the potential of these to affect symptomatology.

The patients who fulfilled the above criteria and had normal physical examination were considered to undergo screening investigations $(\mathrm{Hb} \%, \mathrm{TC}, \mathrm{DC}, \mathrm{ESR}$, blood glucose, serum $\mathrm{TSH}$, stool for $\mathrm{R} / \mathrm{M} / \mathrm{E}$ and short colonoscopy or double contrast barium enema) to exclude organic disease.

\section{Study design}

In this study, a total of 80 patients were included. IBS may be diarrhea predominant or constipation predominant. In order to administer the same pharmacological treatment only diarrhea predominant patients without any organic disease were recruited for the present study. Operationally, the diarrhea was defined as the patient's self-described passage of loose, soft stool and more than 3 motions/day. The patients randomly allocated to a group consisting of 40 patients (male 30 , female 10) who were given only pharmacological management with Mebeverine Hydrochloride $135 \mathrm{mg}$ thrice daily half an hour before meal and Amitryptyline $10 \mathrm{mg}$ at night for 6 months, and the other 40 patients (male 29 and female 11) were given education by a structured and planned educational class for $1 \mathrm{hr}$ in addition to the same pharmacological treatment. The emphasis of the class was to provide general education about IBS and healthy lifestyle modifications in the areas of diet, stress management and exercise, as well as appropriate use of medications. ${ }^{9,10}$ 
At the end of the class, the patients were provided with a leaflet containing the sum and substance of the class in Bengali so that patients can read the information again, understand it thoroughly and apply the rules and get improved. For stress management, all the 40 patients of education group were sent to the psychiatry department of BSMMU to attend the relaxation therapy.

Recruited patients were literate and were willing to come for weekly follow-up for first 1 month and the last follow-up after 6 months. Informed consent was taken. All patients were symptomatic at the time of inclusion period.

\section{Assessment of improvement}

In both groups, changes of symptoms and quality of life of the patients were assessed by using a valid IBS related quality of life (IBS-QOL) instrument. ${ }^{11,12}$ Symptom scoring of IBS included four major symptoms: abdominal pain, stool frequency, stool form and flatulence. Symptom score was applied before starting treatment at baseline and weekly for four weeks. A doctor on the basis of weekly interview did this scoring. A Bengali version QOL instrument was used which was previously used by the department of Gastroenterology, BSMMU. Bengali version IBS-QOL was given to each patient before the start of treatment, 1 month after treatment and after 6 months i.e. at the end of study pried. Patients themselves scored on IBS-QOL instrument. Improvement was assessed by changes of symptoms in symptoms scoring system and changes of score in IBS-QOL instrument.

\section{Data processing and analysis}

The chi-squared test was used to examine the difference between groups in case of symptom analysis. The difference between the two groups was examined by unpaired $t$-test and paired $t$-test in the same group before and after treatment to see the change in quality of life.
Results

Age range of the patients was 16 to 60 years. The mean age (SD) of education group was 28.7 (9.8) years whereas the mean age (SD) of without education group was 27.9 (5.6) years. There was no significant difference between the patient groups for any key demographic features. Total of nine patients dropped out from this study, three from the education group and six from the without education group.

There was no significant difference in severity of symptoms between drug treatment group (score 119.0) and education plus drug treatment group (score 119.6) at the baseline. Seventy one patients completed the trial. At baseline, 37 patients of the education group had pain score varying from 5-10 points. Even after one week from the start, the pain score was similar. In subsequent weeks, there was variation in improvement of abdominal pain score. At the end of four weeks $10(27 \%)$ patients had marked improvement in abdominal pain and were varying from 0-4 points but the remainder 27 (83\%) patients had no change in the abdominal pain. In the group without education, the scenario of experiencing pain was similar to that of education group as all 34 patients had pain score range between 5-10 points. However, at the end of four weeks, $7(20 \%)$ had pain score in the range of $0-4$ and the rest of the $27(80 \%)$ patients remained as before. The difference of improvement between the education group and without education group was not statistically significant, though the subsidence of pain in both groups before and after treatment and/or education was statistically significant $(p<0.01)$.

On the other hand, regarding the bowel movement at the end of first week, all the patients of both the study groups had stool frequency of more than 3 times/day more than $50 \%$ of time at baseline. In the education group at the end of first week of treatment, $5(13 \%)$ patients had less frequent motions i.e. more than 3 times/day, whereas $4(12 \%)$ patients of without education group 
achieved this improvement. At the end of 4 weeks treatment, $13(35 \%$ and 38\%, respectively) patients in each group had less frequent motions. The improvement of stool frequency was significant in both the groups before and after treatment $(p<0.001)$, but the difference of improvement between the two groups was not significant.

Regarding the consistency of stool, in the group with education, $2(5 \%)$ patients had less frequent (i.e. up to $25 \%$ of baseline frequency) soft stool but none of the group without education at the end of one week treatment. There was variation in improvement in subsequent weeks. At the end of four weeks 15 (40\%) patients in the group with education and $13(38 \%)$ patients in the group without education group had marked improvement in stool consistency. The remaining $22(60 \%)$ and 21 (62\%), respectively were unchanged. The difference in improvement in both groups, before and after treatment was highly significant $(p<$ 0.001), but the difference between the groups was not significant.

Among the 37 patients in the group with education, $22(60 \%)$ had frequent flatulence and the remaining 15 (40\%) had less frequent flatulence. There was variation in improvement in subsequent weeks of treatment. At the end of four weeks, 19 $(51 \%)$ had less frequent and the rest 18 $(49 \%)$ had frequent flatulence. Similarly in the group without education, $12(36 \%)$ and $22(64 \%)$ patients had less frequent and frequent flatulence, receptively before treatment. At the end of four weeks, this figure was 13 (38\%) and 21 (62\%), respectively. The improvement of flatulence was not significant in either group and between groups.

Regarding quality of life score, in the group with education, the mean score before treatment was 119.5 and in the group without education group it was 118.6. One month after treatment the mean score was 83.5 and 84.6, respectively. The improvement in QOL score at 1 month and 6 months after treatment in the both study groups was highly significant $(p<0.001)$, but the difference in improvement between the two groups both at 1 month and 6 month was not statistically significant.

\section{Discussion}

IBS is the most common functional disorder of the alimentary tract in which bowel habits are altered in association with abdominal pain or discomfort, has a prevalence of $12 \%$ among adults in the United States and similar prevalence worldwide. ${ }^{13}$ As the pathophysiology of IBS in not clearly understood, it is difficult to treat. But effective management may lessen the symptoms of IBS and may lead to improvement in QOL. Several groups of drugs have been used in the treatment of IBS like bulking agents, antidiarrheal, anti-spasmodics, anti-depressants, etc. ${ }^{6}$ As IBS symptoms can be elucidated or exacerbated by diet or stress, patient education may be beneficial in managing their symptoms. ${ }^{7}$ However, a little is known about its role in management of IBS. ${ }^{10}$ The degree to which patient education in the areas of diet, exercise and stress management can improve symptoms of IBS is unknown. ${ }^{14}$ Several studies have shown that education is effective in relieving symptoms and improving well-being in IBS patients. ${ }^{15,16}$ On the other hand, there are some other studies which failed to show significant benefit from educational class. ${ }^{10,16}$ There is no study to see the effects of education on IBS patients in Bangladesh. This study was conducted to observe the effect of education on IBS patients. Seventy one (57 male and 14 female) patients were included in this study (37 patients in group with education and 34 in group without education). Although the prevalence of IBS is higher in females, in this study female patients are less in number as they are reluctant to attend hospital and some also denied being included in the trial.

In this study, abdominal pain improved after one month of treatment in $27 \%$ of the group with education and $20 \%$ of the without education group. In a study by Colwell and others showed that pain decreased significantly at one month after education, in 
which the pain score came down from 3 (baseline) to 2.4 (after 1 month) on a scale $0-4 .{ }^{14}$ In that study at baseline and at 1 month, there were significant associations between exercise and pain, more exercise was associated with less pain. On the contrary, in another study the pain severity scores decreased both for class attendees and non attendees; therefore, class attendance did not predict pain improvement by univariate and multivariate linear regression. ${ }^{10}$

In this study, only diarrhea predominant patients were included. All the patients had stool frequency $>3$ times/day and soft stool. At the end of one month of treatment improvement of diarrhea was $35 \%$ in group with education and $38 \%$ in the without education group. Stool consistency improvement was $40 \%$ and $38 \%$, respectively. Improvement occurred in both groups but difference was not statistically significant between the two groups. In one study with a $3 \mathrm{hr}$ educational class, there was a $57 \%$ improvement in diarrhea at 1 month after treatment than baseline. This improvement was $71 \%$ at 6 months after the onset of treatment.

In our study, $60 \%$ patients in the group with education and $64 \%$ patients in the group without education had troublesome flatulence. After 4 weeks of treatment, the number of patients with flatus came down to some extent $(49 \%)$ in the group with education but the number also went down in the group without education (62\%). This improvement was not statistically significant. In a study in the United States, $77 \%$ of the patients had bloating at baseline but at the end of 1 month of treatment only $46 \%$ had bloating. ${ }^{14}$ This study also showed a significant number of patients reporting no bloating of distension at 6 months. In these patients, nausea was also less at 1 month and 6 months and heart burn was less at 1 month. In anther study delivering IBS class consisting of 5 weekly $2 \mathrm{hr}$ sessions of education on IBS on a biological mind body disease model emphasizing self efficacy (diet, medication, coping style) and practical instructions in simple relaxation techniques, the outcome measures at baseline and at 3 months showed an improvement in global severity of symptoms. ${ }^{18}$

IBS-QOL instrument was used as a parameter of improvement in this trial. The results showed that significant improvement occurred in both the groups of patients at 1 month and 6 months in respect to their baseline IBS-QOL score. But the improvement was not significantly higher in the group with education in comparison to the group without education.

\section{Conclusion}

This study was conducted to observe the effect of education on IBS patients. The IBS-QOL instrument was used as a parameter of improvement in this trial. It showed that significant improvement occurred at 1 month and 6 months in respect to their baseline IBS-QOL score in both the groups of patients with and without educational class before the start of the study. But the improvement was not significantly higher in the group with education in comparison to the group without education. Therefore, it can be concluded that the educational class had no extra impact on drug treatment protocol for IBS used in the present study.

\section{References}

1. Thomson WG, Greed F, Drossman DA, Heaton KW, Mazzacea G, Functional bowel disorders and functional abdominal pain. Gastroenterol Int 1992;5:75-91.

2. Talley NJ. Functional gastrointestinal disorders. In: Freidman SL, Mc Quaid $\mathrm{KR}$, Grendell JH. Editors. Current diagnosis and treatment in gastroenterology. New York, 2003;pp.97-107.

3. Masud MA, Hasan M, Khan AK. Irritable bowel syndrome in a rural community in Bangladesh: prevalence, symptom pattern and health seeking behavior. Am J Gastroenterol 2001;96:1547-52.

4. Lynn BR, Friedman SL. Irritable bowel syndrome. N Eng J Med 1993; 329:1910-45. 
5. Havdenak N. Loperamide treatment of the irritable bowel syndrome. Scan J Gastroenterol 1987;3:153-6.

6. Klein KB. Controlled treatment trial in irritable bowel syndrome: a critique. Gastroenterology 1998;95:234-41.

7. Drossman DA, Camilleri M, Mayer EA, Whitehead WE. AGA technical review on irritable bowel syndrome. Gastroenterology 2002;123:2108-31.

8. Cantor JC, Morisky DE, Green LW, Levine DM, Salkever DS. Costeffectiveness of educational interventions to improve patient outcomes in blood pressure control. Prev Med 1985; 14:782-800.

9. Shaw G, Srivastava ED, Sadlier M, Swann P, James JH, Rhodes J. Stress management for irritable bowel syndrome: controlled trial. Digestion 1991;50:36-42.

10. Saito YA, Prather CM, Carol T, et al. Effects of multidisciplinary education on outcomes in patients with IBS. Clin Gastroenterol Hepatol 2004;2:576-84.

11. Read NW. Review in depth irritable bowel syndrome. Eur J Gastroenterol Hepatol 1994;6:457-89.

12. Drossman DA, Patrik DL, Whitehead WE, et al. Further validation of the IBS-QOL: a disease specific quality of life questionnaire. Am J Gastroenterol 2000;95:999-1007.

13. Mertz HR. Irritable bowel syndrome. N Eng J Med 2003;349:2136-48.

14. Colwell LJ, Prather CM, Philips SF, Zienmeister AR. Effects of an irritable bowel syndrome educational class on health promotion behaviors and symptoms. Am J Gastroenterol 1998;93,901-5.

15. Weerdt I, Visser AP, Kok GJ, et al. Randomized controlled multicentre evaluation of an education programme for insulin-treated diabetic patients: effects on metabolic control, quality of life, and costs of therapy. Diabet Med 1991;8:338-45.

16. Bennet $P$, Wilkinson S. A comparison of psychological and medical treatment of irritable bowel syndrome. $\mathrm{Br} J$ Clin Psychol 1985;24:215-6.

17. Schwarz SP, Blanchard EB, Neff DF. Behavioral treatment of irritable bowel syndrome: 1 year follow up study. Biofeedback Self Regul 1986;11:189-98.

18. Labus J, Gupta A, Gill HK, et al. Randomised clinical trial: symptoms of the irritable bowel syndrome are improved by a psycho-education group intervention. Aliment Pharmacol Ther 2013;37:304-15.

\section{Suggestion for citation of the above:}

Paik B, Sarker SK, Chowdhury PK, Ahmed MS. A comparison of drug treatment outcome of irritable bowel syndrome patients with and without education: a randomized controlled study. Mediscope 2015;2(2):22-7. 